\title{
Eta-Conversion for the Languages of Explicit Substitutions
}

\section{Thérèse Hardin}

LITP, Université Paris 6, 4 Place Jussieu, 75252 Paris Cedex 05 and INRIA-Rocquencourt, Domaine de Voluceau, BP 105, 78153 Le Chesnay Cedex, France-hardin@margaux.inria.fr.

\section{AAECC 5(6), 317-341 (1994)}

In my paper, it is claimed that the system $\lambda \sigma$, augmented with the rewriting relation $\mathrm{c} \eta$, is confluent. This result does not hold. $\lambda \sigma$ and $c \eta$ are confluent. But, these two rewriting relations do not commute: the proposition 5.7 is wrong. However, this proposition remains true on ground terms. So, the correct result is:

Proposition. The system $\lambda \sigma$, augmented with the rewriting relation $\mathrm{c} \eta$, is ground confluent.

In the proof of proposition 5.7, the following case is missing (I label the $\lambda$ to ease the explanations).

Let $S$ be a $c \eta$-redex of the form $S \equiv \lambda_{2}\left(\left(\lambda_{1} a_{1}\right) 1\right)$. By definition, $\lambda_{1} a_{1}={ }_{\sigma} b[\uparrow]$ so $b=\lambda_{1}\left(b_{1}\right)$ and $a_{1}=b_{1}[\Uparrow(\uparrow)]$. Now, let $R=\left(\lambda_{1} a_{1}\right) 1$. We have to prove that sim $\eta$ and $\operatorname{sim} \beta$ commute.

$$
R \stackrel{\text { (Beta) }}{\longrightarrow} a_{1}[1 . i d] \text { and } S \equiv \lambda_{2}\left(\left(\lambda_{1} a_{1}\right) 1\right) \stackrel{\text { (Beta) }}{\longrightarrow} \lambda_{2}\left(a_{1}[1 \cdot i d]\right) . \text { Now, } S \stackrel{\text { c }}{\longrightarrow} \lambda_{1}\left(b_{1}\right) .
$$

We have to prove that $a_{1}[1 \cdot \mathrm{id}]={ }_{\sigma} b_{1}$, so to prove that $b_{1}[\uparrow(\uparrow)][1 \cdot i d]={ }_{\sigma} b_{1}$. But, $b_{1}[\Uparrow(\uparrow)][1 \cdot i d]={ }_{s} b_{1}[1 \cdot \uparrow]$ and it is not true in general that $b_{1}={ }_{s} b_{1}[1 \cdot \uparrow]$. However, this equality remains true if $b_{1}$ is a ground term, by applying the interpretation method. 